Bull. Austral. Math. Soc.

Vor. $42(1990) \quad[407-416]$

\title{
A DISTANCE FOR SIMLARITY CLASSES OF SUBMANIFOLDS OF A EUCLIDEAN SPACE
}

\author{
Patrizio Frosini
}

\begin{abstract}
A distance is defined on the quotient of the set of submanifolds of a Euclidean space, with respect to similarity. It is then related to a previously defined function which captures the metric behaviour of paths.
\end{abstract}

\section{INTRODUCTION}

In the long-term project of formalising the intuitive concept of "shape" of an object $[5,6]$, a natural problem is to define a distance which vanishes for similar objects. This is the aim of the present paper, in which we define a distance between equivalence classes of manifolds embedded in $E^{m}$, with respect to similarity (Section 1 ). A result connecting this distance with a function defined in [5] is presented in Section 2.

The distance is defined very much in the way Teichmüller distance is, the essential difference being that the latter considers a ratio between a maximum and a minimum at each point, while ours uses the ratio between a global maximum and a global minimum. This is due to the necessity of using a distance that calls attention not to conformal mappings but to similarities, because of the central position that similarities hold in building the intuitive concept of shape.

\section{ThE BASIC DEFINITION}

The proposal of this section is to define a distance on the quotient set of the $C^{\infty}$ compact $n$-manifolds embedded in the Euclidean space $E^{m}$ with respect to the relation of similarity. In one sense this distance will measure how different the shapes of two manifolds are. The definition of this distance will be very similar to the well-known one of Teichmüller distance (see $[8,2])$.

Definition 1.1: Let $\Sigma_{n}$ be the set of the $C^{\infty}$ compact $n$-manifolds $(n>0)$ without boundary and embedded in the Euclidean space $E^{m}$ and let $R$ be the equivalence relation so defined: for every $\mathcal{M}, \mathcal{N} \in \Sigma_{n} \mathcal{M R N}_{R}$ if and only if there exists a similarity

Received 5 December 1989

Work performed under the auspicies of the G.N.S.A.G.A. of the C.N.R. and within the Project "Geometria delle Varietz Differenziabili" of the M.U.R.S.T. (Italy), under the supervision of Professor M. Ferri.

Copyright Clearance Centre, Inc. Serial-fee code: 0004-9729/90 \$A2.00+0.00. 
transformation $\theta: E^{m} \rightarrow E^{m}$ such that $\theta(\mathcal{M})=\mathcal{N}$. The equivalence class to which the manifold $\mathcal{M}$ belongs will be denoted by $[\mathcal{M}]$.

For every pair $(\mathcal{M}, \mathcal{N})$ of $n$-manifolds in $\Sigma_{n}$ let us denote by $D(\mathcal{M}, \mathcal{N})$ the set of the $C^{\infty}$ diffeomorphisms $\varphi$ from $\mathrm{E}^{m}$ to $\mathrm{E}^{m}$ such that $\varphi(\mathcal{M})=\mathcal{N}$ and $\varphi$ and $\varphi^{-1}$ have differentials with bounded norm. The norm which we consider is the usual one for linear operators (see [4], Part 1, Chapter II, Section 3) and for every linear operator $A$ we shall denote its norm by $\|A\|$. On the quotient set $\Sigma_{n} / \mathcal{R}$ let us define the following function $\sigma$ with values in $\mathrm{R} \cup\{+\infty\}$ :

$$
\sigma([\mathcal{M}],[\mathcal{N}])=\left\{\begin{array}{lc}
+\infty & \text { if } D(\mathcal{M}, \mathcal{N})=\emptyset \\
\inf _{\varphi \in D(\mathcal{M}, \mathcal{N})}\left\{\log \left(\sup _{P \in E^{m}}\|d \varphi(P)\| \cdot \sup _{Q \in E^{m}}\left\|d \varphi^{-1}(Q)\right\|\right)\right\} & \text { otherwise }
\end{array}\right.
$$

where $d \varphi$ and $d \varphi^{-1}$ are the differentials of $\varphi$ and $\varphi^{-1}$ respectively.

REMARK 1.1. We point out that if $\sigma$ is great (and less than $+\infty$ ) then it means that every $\varphi \in D(\mathcal{M}, \mathcal{N})$ is very different from a similarity transformation because there are points on $\mathcal{M}$ in which the norm of the differential of $\varphi$ has very different values: in other words in order to change $\mathcal{M}$ into $\mathcal{N}$ we must perform a great deformation of $\mathcal{M}$.

In the parallelism between $\sigma$ and Teichmüller distance we point out that in one sense the quotient space $\Sigma_{n} / \mathcal{R}$ and the set $D(\mathcal{M}, \mathcal{N})$ correspond respectively to the Teichmüller space $T_{s}$ and the set of quasiconformal mappings from $\mathcal{M}$ to $\mathcal{N}$. (As regards these last two concepts we refer to [1]).

The definition of $\sigma$ may bring to mind also the well-known concept of Fréchet Distance (see [3], Chapter IX, Section 31.6). In one sense the structures of the two definitions are similar but they arise from quite different ideas.

THEOREM 1.1. The function $\sigma$ is a well-defined distance on $\Sigma_{n} / \mathcal{R}$.

Proof: First of all we observe that $\sigma$ is well-defined. In fact if $\mathcal{M}_{1} R \mathcal{M}_{2}$ and $\mathcal{N}_{1} \mathcal{R} \mathcal{N}_{2}$ (that is $\left[\mathcal{M}_{1}\right]=\left[\mathcal{M}_{2}\right]$ and $\left[\mathcal{N}_{1}\right]=\left[\mathcal{N}_{2}\right]$ ) then there exist two similarity transformations $S_{\lambda}, S_{\mu}: \mathrm{E}^{m} \rightarrow \mathrm{E}^{m}$ whose differentials have everywhere norm $\lambda$ and $\mu$ respectively $(\lambda, \mu \neq 0)$ such that we have $S_{\lambda}\left(\mathcal{M}_{1}\right)=\mathcal{M}_{2}$ and $S_{\mu}\left(\mathcal{N}_{1}\right)=\mathcal{N}_{2}$. Therefore if $D\left(\mathcal{M}_{1}, \mathcal{N}_{1}\right) \neq \emptyset$ we have ( $d$ is the operator that takes a $C^{\infty}$ function to its 
differential):

$$
\begin{aligned}
& \inf _{\varphi \in D\left(\mathcal{M}_{1}, \mathcal{N}_{1}\right)}\left\{\log \left(\sup _{P \in \mathbb{E}^{m}}\|d \varphi(P)\| \cdot \sup _{Q \in E^{m}}\left\|d \varphi^{-1}(Q)\right\|\right)\right\} \\
& =\inf _{\varphi \in D\left(\mathcal{M}_{1}, N_{1}\right)}\left\{\log \left(\left(\frac{\mu}{\lambda} \cdot \sup _{P \in E^{m}}\|d \varphi(P)\|\right) \cdot\left(\frac{\lambda}{\mu} \cdot \sup _{Q \in E^{m}}\left\|d \varphi^{-1}(Q)\right\|\right)\right)\right\} \\
& =\inf _{\varphi \in D\left(\mathcal{M}_{1}, N_{1}\right)}\left\{\log \left(\sup _{R \in E^{m}}\left\|d\left(S_{\mu} \circ \varphi \circ S_{\lambda}^{-1}\right)(R)\right\| \cdot \sup _{T \in E^{m}}\left\|d\left(S_{\mu} \circ \varphi \circ S_{\lambda}^{-1}\right)^{-1}(T)\right\|\right)\right\} \\
& =\inf _{\psi \in D\left(\mathcal{M}_{2}, N_{2}\right)}\left\{\log \left(\sup _{R \in E^{m}}\|d \psi(R)\| \cdot \sup _{T \in E^{m}}\left\|d \psi^{-1}(T)\right\|\right)\right\} .
\end{aligned}
$$

On the other hand if $D\left(\mathcal{M}_{1}, \mathcal{N}_{1}\right)=\emptyset$ then also $D\left(\mathcal{M}_{2}, \mathcal{N}_{2}\right)=\emptyset$.

These facts prove that the function $\sigma$ is well defined.

In the second place we observe that the following statements are true:

(1) $\sigma([\mathcal{M}],[\mathcal{N}]) \geqslant 0$ for every $\mathcal{M}, \mathcal{N} \in \Sigma_{n}$. In fact if $D(\mathcal{M}, \mathcal{N})=\emptyset$ then $\sigma([\mathcal{M}],[\mathcal{N}])=+\infty>0$ while if $D(\mathcal{M}, \mathcal{N}) \neq \emptyset$ then for every $\varphi \in D(\mathcal{M}, \mathcal{N})$ we have:

$$
\begin{gathered}
\log \left(\sup _{P \in \mathbb{E}^{m}}\|d \varphi(P)\| \cdot \sup _{Q \in E^{m}}\left\|d \varphi^{-1}(Q)\right\|\right) \geqslant \log \left(\sup _{P, Q \in E^{m}}\left\|d \varphi(P) \circ d \varphi^{-1}(Q)\right\|\right) \\
\geqslant \log \left(\sup _{P \in E^{m}}\left\|d \varphi(P) \circ d \varphi^{-1}(\varphi(P))\right\|\right)=\log \left(\sup _{P \in E^{m}}\|I\|\right)=\log (1)=0
\end{gathered}
$$

(where $I$ is the identity linear operator).

(2) $\sigma([\mathcal{M}],[\mathcal{M}])=0$ for every $\mathcal{M} \in \Sigma_{n}$. In fact if we take $\varphi$ equal to the identity function we have that $\log \left(\sup _{P \in \mathrm{E}^{m}}\|d \varphi(P)\| \cdot \sup _{Q \in \mathrm{E}^{m}}\left\|d \varphi^{-1}(Q)\right\|\right)=\log (1)=0$. The thesis follows from this fact and the previous statement 1.

(3) $\sigma([\mathcal{M}],[\mathcal{N}])=0$ implies that $[\mathcal{M}]=[\mathcal{N}]$. We can prove this statement in the following way: if $\sigma([\mathcal{M}],[\mathcal{N}])=0$ then there exists a sequence $\left\{\varphi_{i}\right\}_{i \in N}$ of diffeomorphisms in $D(\mathcal{M}, \mathcal{N})$ such that $\lim _{i \rightarrow+\infty} \log \left(\sup _{P \in E^{m}}\left\|d \varphi_{i}(P)\right\| \cdot \sup _{Q \in E^{m}}\left\|d \varphi_{i}^{-1}(Q)\right\|\right)=0$, that is $\lim _{i \rightarrow+\infty} \sup _{P \in E^{m}}\left\|d \varphi_{i}(P)\right\| \cdot \sup _{Q \in E^{m}}\left\|d \varphi_{i}^{-1}(Q)\right\|=1$. For the sake of simplicity we shall denote $\sup _{P \in \mathbb{E}^{m}}\left\|d \varphi_{i}(P)\right\|$ by $M_{i}$ and $1 / \sup _{Q \in E^{m}}\left\|d \varphi_{i}^{-1}(Q)\right\|$ by $m_{i}$ (we point out that because of the definition of $D(\mathcal{M}, \mathcal{N})$ it follows that $\left.0<m_{i}, M_{i}<+\infty\right)$.

By observing that for every $P, Q \in \mathrm{E}^{m}$ (and in particular for every $P, Q \in \mathcal{M}$ ) and $i \in N$ it follows that $m_{i} \cdot\|P-Q\| \leqslant\left\|\varphi_{i}(P)-\varphi_{i}(Q)\right\| \leqslant M_{i} \cdot\|P-Q\|$, and calling $\Delta(\mathcal{M})$ and $\Delta(\mathcal{N})$ respectively the diameters of $\mathcal{M}$ and $\mathcal{N}$, we obtain $m_{i} \cdot \Delta(\mathcal{M}) \leqslant$ $\Delta(\mathcal{N}) \leqslant M_{i} \cdot \Delta(\mathcal{M})$. Therefore we can say that there exist two values $h, k \in \mathbb{R}$ such that $0<h \leqslant M_{i}$ and $0<m_{i} \leqslant k$ for every $i \in N$, otherwise the manifolds $\mathcal{M}$ and 
$\mathcal{N}$ could not both have a finite nonvanishing diameter, against the hypothesis that $\mathcal{M}$ and $\mathcal{N}$ are compact $n$-dimensional $(n>0)$ manifolds.

In particular (if necessary by extracting a subsequence) we can suppose that $\left\{m_{i}\right\}_{i \in N}$ converges to a finite value $\bar{m}$. Since $\lim _{i \rightarrow+\infty} M_{i} / m_{i}=1$ we have that also the sequence $\left\{M_{i}\right\}_{i \in N}$ converges to the value $\bar{m}$, and this fact implies that $\bar{m} \geqslant h>0$ and that all the functions $\varphi_{i}$ have differentials with norms everywhere bounded by a constant $c<+\infty$ : so the functions $\varphi_{i}$ are equiuniformly continuous. Moreover, if for every $j \in N, K_{j}$ is a compact subset of $E^{m}$ of diameter $D_{j}$ containing $\mathcal{M}$ and $\bigcup_{j \in N} K_{j}=E^{m}$, then for every $i \in N \varphi_{i}\left(K_{j}\right)$ is contained in the compact set $B_{K_{j}}$ made up by the points of $E^{m}$ distant from $\mathcal{N}$ less than $D_{j} \cdot c$. So for every such compact $K_{j}$ we can apply the Ascoli-Arzelà generalised theorem to the space of the continuous functions from $K_{j}$ to $B_{K_{j}}$ (see [7], Chapter II, Section 18): this allows us to extract from $\left\{\varphi_{i}\right\}_{i \in N}$ a subsequence $\left\{\varphi_{,(i, j)}\right\}_{i \in N}$ that converges uniformly in $K_{j}$ to a (continuous) function $\bar{\varphi}_{K_{j}}$. Moreover the diagonal subsequence $\left\{\varphi_{s(i, i)}\right\}_{i \in N}$ converges in $E^{m}$ to a (continuous) function $\bar{\varphi}$ extension to $E^{m}$ of the $\bar{\varphi}_{K_{j}}$ 's. For the sake of simplicity in the following we shall use the notation $\left\{\varphi_{i}\right\}_{i \in N}$ instead of $\left\{\varphi_{s(i, i)}\right\}_{i \in N}$.

By computing the limits for $i \rightarrow+\infty$ in the above-mentioned inequality $m_{i} \cdot\|P-Q\| \leqslant\left\|\varphi_{i}(P)-\varphi_{i}(Q)\right\| \leqslant M_{i} \cdot\|P-Q\|$ it follows that $\|\bar{\varphi}(P)-\bar{\varphi}(Q)\|=$ $\bar{m} \cdot\|P-Q\|$ for every $P, Q \in \mathrm{E}^{m}$. Therefore $\bar{\varphi}$ multiplies by $\bar{m}$ all the distances between points and so it is a similarity transformation from $E^{m}$ to $E^{m}(\bar{m} \neq 0)$.

The last thing to prove is that $\bar{\varphi}(\mathcal{M})=\mathcal{N}$. First of all $\bar{\varphi}(\mathcal{M}) \subseteq \mathcal{N}$ : in fact if $P \in$ $\mathcal{M}$ then for every $i \in N \varphi_{i}(P) \in \mathcal{N}$ (since $\varphi_{i} \in D(\mathcal{M}, \mathcal{N})$ ) and so $\bar{\varphi}(P)=\lim _{i \rightarrow+\infty} \varphi_{i}(P)$ belongs to $\mathcal{N}$ because $\mathcal{N}$ is a closed subset of $E^{m}$. In the second place $\bar{\varphi}(\mathcal{M}) \supseteq \mathcal{N}$ : in fact if $Q \in \mathcal{N}$ then $\bar{\varphi}^{-1}(Q) \in \mathcal{M}$ because otherwise $\bar{\varphi}^{-1}(Q)$ would have a distance $\eta>0$ from $\mathcal{M}$ (it is a closed subset of $E^{m}$ ). In such a case, since $\varphi_{i}$ multiplies every distance by a number not less than $m_{i}>0, \varphi_{i}\left(\bar{\varphi}^{-1}(Q)\right)$ would have a distance not less than $m_{i} \cdot \eta>0$ from $\varphi_{i}(\mathcal{M})=\mathcal{N}$ and so $Q=\bar{\varphi}\left(\bar{\varphi}^{-1}(Q)\right)=\lim _{i \rightarrow+\infty} \varphi_{i}\left(\bar{\varphi}^{-1}(Q)\right)$ would have a distance not less than $\bar{m} \cdot \eta>0$ from $\mathcal{N}$ : this fact would be against the hypothesis that $Q \in \mathcal{N}$. Therefore $\bar{\varphi}(\mathcal{M})=\mathcal{N}$.

In conclusion $\bar{\varphi}$ is a similarity transformation such that $\bar{\varphi}(\mathcal{M})=\mathcal{N}$ and so we have that $[\mathcal{M}]=[\mathcal{N}]$.

(4) $\sigma([\mathcal{M}],[\mathcal{N}])=\sigma([\mathcal{N}],[\mathcal{M}])$ for every $\mathcal{M}, \mathcal{N} \in \Sigma_{n}$. This equality follows by observing that $\varphi \in D(\mathcal{M}, \mathcal{N})$ if and only if $\varphi^{-1} \in D(\mathcal{N}, \mathcal{M})$ : if $D(\mathcal{M}, \mathcal{N}) \neq \emptyset$ then the infima which give $\sigma([\mathcal{M}],[\mathcal{N}])$ and $\sigma([\mathcal{N}],[\mathcal{M}])$ are computed in the same set of values while if $D(\mathcal{M}, \mathcal{N})=\emptyset$ then also $D(\mathcal{N}, \mathcal{M})=\emptyset$ and the equality is obvious.

(5) $\sigma([\mathcal{L}],[\mathcal{N}]) \leqslant \sigma([\mathcal{L}],[\mathcal{M}])+\sigma([\mathcal{M}],[\mathcal{N}])$ for every $\mathcal{L}, \mathcal{M}, \mathcal{N} \in \Sigma_{n}$. We can prove this in the following way. If $\sigma([\mathcal{L}],[\mathcal{M}])=+\infty$ or $\sigma([\mathcal{M}],[\mathcal{N}])=+\infty$ then 
$\sigma([\mathcal{L}],[\mathcal{M}])+\sigma([\mathcal{M}],[\mathcal{N}])=+\infty \geqslant \sigma([\mathcal{L}],[\mathcal{N}])$. On the other hand if $\sigma([\mathcal{L}],[\mathcal{M}])<$ $+\infty$ and $\sigma([\mathcal{M}],[\mathcal{N}])<+\infty$ then $D(\mathcal{L}, \mathcal{M}) \neq \emptyset$ and $D(\mathcal{M}, \mathcal{N}) \neq \emptyset$ and it follows that the functions in $D(\mathcal{L}, \mathcal{N})$ are all and only those which are obtained as a composition of a function in $D(\mathcal{L}, \mathcal{M})$ with a function in $D(\mathcal{M}, \mathcal{N})$ (so $D(\mathcal{L}, \mathcal{N}) \neq \emptyset$ ). Therefore in this second case we have:

$$
\begin{aligned}
& \sigma([\mathcal{L}],[\mathcal{M}])+\sigma([\mathcal{M}],[\mathcal{N}])=\inf _{\varphi \in D(\mathcal{L}, \mathcal{M})}\left\{\log \left(\sup _{P \in E^{m}}\|d \varphi(P)\| \cdot \sup _{Q \in E^{m}}\left\|d \varphi^{-1}(Q)\right\|\right)\right\} \\
& +\inf _{\forall \in D(\mathcal{M}, \mathcal{N})}\left\{\log \left(\sup _{Q \in E^{m}}\|d \psi(Q)\| \cdot \sup _{R \in E^{m}}\left\|d \psi^{-1}(R)\right\|\right)\right\} \\
& =\inf _{\substack{\varphi \in D(\mathcal{L}, \mathcal{M}) \\
\nu \in D(\mathcal{M}, \mathcal{N})}}\left\{\log \left(\sup _{P \in E^{m}}\|d \varphi(P)\| \cdot \sup _{Q \in E^{m}}\left\|d \varphi^{-1}(Q)\right\|\right)\right. \\
& \left.+\log \left(\sup _{Q \in E^{m}}\|d \psi(Q)\| \cdot \sup _{R \in E^{m}}\left\|d \psi^{-1}(R)\right\|\right)\right\} \\
& =\inf _{\substack{\varphi \in D(\mathcal{L}, \mathcal{M}) \\
\psi \in D(\mathcal{M}, \mathcal{N})}}\left\{\log \left(\sup _{P \in \mathbb{E}^{m}}\|d \varphi(P)\| \cdot \sup _{Q \in E^{m}}\left\|d \varphi^{-1}(Q)\right\| \cdot \sup _{Q \in E_{m}^{m}}\|d \psi(Q)\| \cdot \sup _{R \in \mathbb{E}^{m}}\left\|d \psi^{-1}(R)\right\|\right)\right\} \\
& \geqslant \inf _{\substack{\varphi \in D(\mathcal{L}, \mathcal{M}) \\
\psi \in D(\mathcal{M}, \mathcal{N})}}\left\{\log \left(\sup _{\boldsymbol{P} \in \mathbf{E}^{m}}(\|d \psi(\varphi(P))\| \cdot\|d \varphi(P)\|) \cdot \sup _{\boldsymbol{R} \in \mathbf{E}^{m}}\left(\left\|d \varphi^{-1}\left(\psi^{-1}(R)\right)\right\| \cdot\left\|d \psi^{-1}(R)\right\|\right)\right)\right\} \\
& \geqslant \inf _{\substack{\varphi \in D(\mathcal{L}, \mathcal{M}) \\
\phi \in D(\mathcal{M}, \mathcal{N})}}\left\{\log \left(\left(\sup _{P \in E_{m}}\|d \psi(\varphi(P)) \circ d \varphi(P)\|\right) \cdot\left(\sup _{R \in E^{m}}\left\|d \varphi^{-1}\left(\psi^{-1}(R)\right) \circ d \psi^{-1}(R)\right\|\right)\right)\right\} \\
& =\inf _{\substack{\varphi \in D(\mathcal{L}, \mathcal{M}) \\
\psi \in D(\mathcal{M}, \mathcal{N})}}\left\{\log \left(\left(\sup _{P \in E^{m}}\|d(\psi \circ \varphi)(P)\|\right) \cdot\left(\sup _{R \in E^{m}}\left\|d(\psi \circ \varphi)^{-1}(R)\right\|\right)\right)\right\} \\
& =\inf _{\theta \in D(\mathcal{C}, \mathcal{N})}\left\{\log \left(\left(\sup _{P \in E^{m}}\|d \theta(P)\|\right) \cdot\left(\sup _{R \in E^{m}}\left\|d \theta^{-1}(R)\right\|\right)\right)\right\}=\sigma([\mathcal{L}],[\mathcal{N}])
\end{aligned}
$$

The above-mentioned properties show that $\sigma$ is a distance on $\Sigma_{n} / \mathcal{R}$.

\section{A result about the distance $\sigma$}

The above-defined distance is difficult to compute and we need some tools to find information about the distance between two given equivalence classes $[\mathcal{M}]$ and $[\mathcal{N}]$ without really studying all the diffeomorphisms in $D(\mathcal{M}, \mathcal{N})$. For this reason we give in this section a result that provides a lower bound for $\sigma$.

First of all we need to define the function $f_{1}^{L}(\mathcal{M}, x, y)$ in the real variables $x, y$. For every manifold $\mathcal{M} \in \Sigma_{n}$ and every $x, y \in R$ we consider the set $P(\mathcal{M}, x)$ of the piecewise $C^{1}$ closed paths in $\mathcal{M}$ of length less than or equal to $x$ and we quotient it with respect to the following equivalence relation $\underset{y}{\frac{L}{y}}$ : for every $\alpha, \beta \in P(\mathcal{M}, x)$ we 
have $\alpha \frac{L}{y} \beta$ if and only if either $\alpha$ and $\beta$ are the same path or there exists a homotopy $H_{\alpha \beta}(t, \tau)$ between $\alpha$ and $\beta$ such that for every $\tau \in[0,1]$ the path in the variable $t H_{\alpha \beta}(t, \tau)$ belongs to $\mathrm{P}(\mathcal{M}, y)$, that is we can "transform with continuity $\alpha$ into $\beta$ without exceeding the length $y$ ". $H_{\alpha \beta}$ will be said to be a $y$ - $L$-homotopy. We shall define $f_{1}^{L}(\mathcal{M}, x, y)$ as the number of equivalence classes into which $P(\mathcal{M}, x)$ is divided by the relation of $y$ - $L$-homotopy (we shall denote all the cardinalities $\geqslant \aleph_{0}$ by the unique symbol " $+\infty$ ").

Of course if $x \geqslant 0$ and $x>y$ then $f_{1}^{L}(\mathcal{M}, x, y)=+\infty$ and if $x<0$ then $f_{1}^{L}(\mathcal{M}, x, y)=0$. For a more precise and detailed definition of the function $f_{1}^{L}(\mathcal{M}, x, y)$ we refer to the paper [5], Section2.

By using the following results we shall point out that even a partial knowledge of the functions $f_{1}^{\mathrm{L}}(\mathcal{M}, \cdot, \cdot)$ and $f_{1}^{\mathrm{L}}(\mathcal{N}, \cdot, \cdot)$ provides a lower bound for $\sigma([\mathcal{M}],[\mathcal{N}])$.

Lemma 2.1. Let $\mathcal{M}, \mathcal{N} \in \Sigma_{n}$ and $\varphi \in D(\mathcal{M}, \mathcal{N})$. Then for every $k, h \in \mathbf{R}^{+}$ such that $\sup _{P \in E^{m}}\|d \varphi(P)\| \leqslant k$ and $\sup _{Q \in \mathbb{E}^{m}}\left\|d \varphi^{-1}(Q)\right\| \leqslant 1 / h$ and every $x, y, \xi, \eta \in \mathbf{R}$ with $\xi \geqslant k x$ and $\eta \leqslant h y$ we have $f_{1}^{\mathrm{L}}(\mathcal{N}, \xi, \eta) \geqslant f_{1}^{\mathrm{L}}(\mathcal{M}, x, y)$.

ProOF: We observe that because of its definition the function $f_{1}^{L}(\mathcal{N}, \xi, \eta)$ is nondecreasing in the variable $\xi$ and non-increasing in the variable $\eta$, therefore it will suffice to prove that $f_{1}^{\mathrm{L}}(\mathcal{N}, k x, h y) \geqslant f_{1}^{\mathrm{L}}(\mathcal{M}, x, y)$. In order to prove this we shall construct an injective mapping $F$ from $\mathrm{P}(\mathcal{M}, x) / \underset{y}{\stackrel{L}{=}}$ to $\mathrm{P}(\mathcal{N}, k x) / \underset{h y}{\stackrel{L}{=}}$ (we shall suppose that $x \geqslant$ 0 and therefore that $\mathrm{P}(\mathcal{M}, x) / \underset{\bar{y}}{\stackrel{L}{y}}$ and $\mathrm{P}(\mathcal{N}, k x) / \underset{h_{y}}{\stackrel{L}{=}}$ are not empty because otherwise $f_{1}^{\mathrm{L}}(\mathcal{N}, k x, h y)=f_{1}^{\mathrm{L}}(\mathcal{M}, x, y)=0$ and the thesis is trivial). This fact will imply that the cardinality of $\mathbf{P}(\mathcal{M}, x) / \underset{y}{\frac{L}{y}}$ is less than or equal to the cardinality of $P(\mathcal{N}, k x) / \frac{L}{h y}$ : since by definition of $f_{1}^{L}$ we have $f_{1}^{L}(\mathcal{M}, x, y)$ equal to the number of equivalence classes in $\mathbf{P}(\mathcal{M}, x) / \underset{y}{\stackrel{L}{y}}$ and $f_{1}^{L}(\mathcal{N}, k x, h y)$ equal to the number of equivalence classes in $P(\mathcal{N}, k x) / \frac{L}{=}$, the thesis of the lemma will follow immediately.

Now let us construct $F$. For every equivalence class $C \in \mathrm{P}(\mathcal{M}, x) / \stackrel{L}{y}$ let us fix arbitrarily one path $\alpha$ in $C$. We observe that $\varphi \circ \alpha$ belongs to $\mathrm{P}(\mathcal{N}, k x)$ because of the inequality $\sup _{P \in \mathbb{E}^{m}}\|d \varphi(P)\| \leqslant k$. Define $F(C)$ as the equivalence class of $\varphi \circ \alpha$ in $\mathrm{P}(\mathcal{N}, k x) / \underset{h_{y}}{\stackrel{L}{=}}$.

If $F\left(C_{1}\right)=F\left(C_{2}\right)$ then, calling $\alpha$ and $\beta$ the respective representatives of $C_{1}$ and $C_{2}$, we have by definition that either $\tilde{\alpha}=\varphi \circ \alpha$ and $\tilde{\beta}=\varphi \circ \beta$ are the same path or there exists an $h y$-L-homotopy $\tilde{H}_{\tilde{\alpha} \tilde{\beta}}$ between $\tilde{\alpha}$ and $\tilde{\beta}$. In the first case we have that 
$\alpha$ and $\beta$ are the same path and therefore that $C_{1}=C_{2}$. In the second case, because of the hypothesis $\sup _{Q \in E^{m}}\left\|d \varphi^{-1}(Q)\right\| \leqslant 1 / h$ we have that the function $H_{\alpha \beta}=\varphi^{-1} \circ \widetilde{H}_{\tilde{\alpha} \tilde{\beta}}$ is a $y$ - $L$-homotopy between $\alpha$ and $\beta$ and so $C_{1}=C_{2}$ (incidentally we observe that because of the choice of only one representative per class this equality implies that $\alpha$ and $\beta$ are the same path also in this case). Therefore in every case $C_{1}=C_{2}$ and so $F$ is injective.

Theorem 2.1. Let $\mathcal{M}, \mathcal{N} \in \Sigma_{n}$ and let $\Delta(\mathcal{M})$ and $\Delta(\mathcal{N})$ be the diameters of $\mathcal{M}$ and $\mathcal{N}$. If $f_{1}^{\mathrm{L}}(\mathcal{N}, \xi, \eta)<f_{1}^{\mathrm{L}}(\mathcal{M}, x, y)$ with $\xi, \eta, x, y>0$ then $\sigma([\mathcal{M}],[\mathcal{N}]) \geqslant$ $\log (\min \{(\xi \cdot \Delta(\mathcal{M})) /(x \cdot \Delta(\mathcal{N})),(y \cdot \Delta(\mathcal{N})) /(\eta \cdot \Delta(\mathcal{M}))\})$.

Proof: We can suppose $D(\mathcal{M}, \mathcal{N}) \neq \emptyset$ because otherwise $\sigma([\mathcal{M}],[\mathcal{N}])=+\infty$ and the thesis is trivial. So let $\varphi \in D(\mathcal{M}, \mathcal{N})$. For sake of simplicity we shall denote $\sup _{P \in \mathbf{E}^{m}}\|d \varphi(P)\|$ by $M$ and $1 / \sup _{Q \in \mathbf{E}^{m}}\left\|d \varphi^{-1}(Q)\right\|$ by $m$. Because of Lemma 2.1 the hypothesis $f_{1}^{\mathrm{L}}(\mathcal{N}, \xi, \eta)<f_{1}^{\mathrm{L}}(\mathcal{M}, x, y)$ implies that either $\xi<M x$ or $\eta>m y$. Therefore either it results $M>\xi / x$ or $1 / m>y / \eta$.

Furthermore, as seen at point (3) of the proof of Theorem 1.1, we have $m \cdot \Delta(\mathcal{M}) \leqslant$ $\Delta(\mathcal{N}) \leqslant M \cdot \Delta(\mathcal{M})$ and so it must follow that $M \geqslant \Delta(\mathcal{N}) / \Delta(\mathcal{M})$ and $(1 / m) \geqslant$ $\Delta(\mathcal{M}) / \Delta(\mathcal{N})$. Therefore if $M>\xi / x$ then we have $M \cdot(1 / m)>(\xi / x) \cdot(\Delta(\mathcal{M}) / \Delta(\mathcal{N}))$ while if $1 / m>y / \eta$ then we have $M \cdot(1 / m)>(\Delta(\mathcal{N}) / \Delta(\mathcal{M})) \cdot(y / \eta)$. In either case we have $M \cdot(1 / m)>\min \{(\xi \cdot \Delta(\mathcal{M})) /(x \cdot \Delta(\mathcal{N})),(y \cdot \Delta(\mathcal{N})) /(\eta \cdot \Delta(\mathcal{M}))\}$. This fact means that for every $\varphi \in D(\mathcal{M}, \mathcal{N})$ the following holds:

$$
\left(\sup _{P \in \mathbb{E}^{m}}\|d \varphi(P)\|\right) \cdot\left(\sup _{Q \in \mathbf{E}^{m}}\left\|d \varphi^{-1}(Q)\right\|\right)>\min \left\{\frac{\xi \cdot \Delta(\mathcal{M})}{x \cdot \Delta(\mathcal{N})}, \frac{y \cdot \Delta(\mathcal{N})}{\eta \cdot \Delta(\mathcal{M})}\right\}
$$

(which is positive for the hypothesis $\xi, \eta, x, y>0$ ). Therefore, because of the definition of $\sigma$, we have that

$$
\sigma([\mathcal{M}],[\mathcal{N}]) \geqslant \log \left(\min \left\{\frac{\xi \cdot \Delta(\mathcal{M})}{x \cdot \Delta(\mathcal{N})}, \frac{y \cdot \Delta(\mathcal{N})}{\eta \cdot \Delta(\mathcal{M})}\right\}\right) .
$$

REMARK 2.1. It is not difficult to reproduce the result of Theorem 2.1 for geodesic diameters instead of Euclidean diameters.

In order to show the usefulness of Theorem 2.1 we give the following example.

EXAMPLE 2.1. Let us consider the manifold $\mathcal{M} \in \Sigma_{2}$ obtained by the rotation in $\mathrm{E}^{3}$, around the $x$-axis, of the curve $\rho=1+4 \cos ^{2}(\theta)$ in $E^{2}$ (expressed in polar coordinates). The curve is shown in Figure 2.1. 


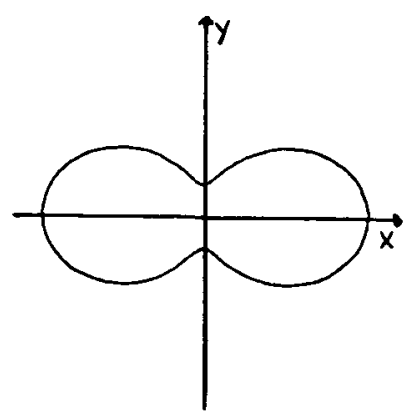

Then let us consider the 2-manifold $\mathcal{N}=S^{2}$, the standard 2-sphere in $\mathrm{E}^{3}$. It is easy to verify that $f_{1}^{L}(\mathcal{M}, 2 \pi, 8) \geqslant 2$ (we cannot change homotopically the closed path of length $2 \pi$ around the central narrowing of $\mathcal{M}$ into a trivial path without exceeding the length 8 during the deformation). On the other hand we have proved in [5] (Proposition 3.1) that $f_{1}^{L}(\mathcal{N}, \xi, \eta)=1$ for every $0 \leqslant \xi \leqslant \eta$. Therefore for $x=2 \pi, y=8, \xi=\eta=4 \sqrt{\pi} / 5$ (these values have been chosen in order to make simpler calculations) we have $f_{1}^{L}(\mathcal{N}, \xi, \eta)<f_{1}^{L}(\mathcal{M}, x, y)$. Moreover $\Delta(\mathcal{M})=10$ and $\Delta(\mathcal{N})=2$. By applying Theorem 2.1 we have $\sigma([\mathcal{M}],[\mathcal{N}]) \geqslant \log (2 / \sqrt{\pi})>0.12$.

REMARK 2.2. It can be useful to look at the construction of the distance $\sigma$ in a more general context. The scheme used here is the following. First of all we consider an equivalence relation $\mathcal{R}$ on a subset $\Omega$ of $\Sigma_{n}$ and then the set $T=\{(\mathcal{M}, \mathcal{N}, \varphi): \mathcal{M}, \mathcal{N} \in$ $\Omega, \varphi \in D(\mathcal{M}, \mathcal{N})\}$.

In the second place we define a non-negative function $\Theta: T \rightarrow R \cup\{+\infty\}$ such that:

(i) for every $\mathcal{M}, \mathcal{N} \in \Omega$ we have $M R \mathcal{N}$ if and only if there exists a sequence $\left\{\varphi_{i}\right\}_{i \in N}$ of diffeomorphisms in $D(\mathcal{M}, \mathcal{N})$ such that $\lim _{i \rightarrow+\infty} \Theta\left(\left(\mathcal{M}, \mathcal{N}, \varphi_{i}\right)\right)=0$;

(ii) for every $\mathcal{M}, \mathcal{N} \in \Omega$ and every $\varphi \in D(\mathcal{M}, \mathcal{N}), \Theta((\mathcal{M}, \mathcal{N}, \varphi))=$ $\Theta\left(\left(\mathcal{N}, \mathcal{M}, \varphi^{-1}\right)\right)$

(iii) for every $\mathcal{L}, \mathcal{M}, \mathcal{N} \in \Omega, \varphi \in D(\mathcal{L}, \mathcal{M})$ and $\psi \in D(\mathcal{M}, \mathcal{N})$ we have $\Theta((\mathcal{L}, \mathcal{M}, \varphi))+\Theta((\mathcal{M}, \mathcal{N}, \psi)) \geqslant \Theta((\mathcal{L}, \mathcal{N}, \psi \circ \varphi))$

In the third place we observe that the function $\sigma([\mathcal{M}],[\mathcal{N}])$ defined equal to $\inf _{\varphi \in D(\mathcal{M}, \mathcal{N})} \Theta((\mathcal{M}, \mathcal{N}, \varphi))$ if $D(\mathcal{M}, \mathcal{N}) \neq \emptyset$ and $+\infty$ otherwise is a distance in $\Omega / \mathcal{R}$.

In this paper we have studied $\sigma$ in the case that $\Omega=\Sigma_{n}, \mathcal{R}$ is the similarity relation and $\Theta((\mathcal{M}, \mathcal{N}, \varphi))=\log \left(\sup _{P \in E^{m}}\|d \varphi(P)\| \cdot \sup _{Q \in E^{m}}\left\|d \varphi^{-1}(Q)\right\|\right)$, but we can change the choice of $\Omega$ and $\mathcal{R}$ and in particular consider other non-negative functions 
$\Theta: T \rightarrow R \cup\{+\infty\}$ satisfying (i), (ii), (iii). For example, for every natural number $k$ with $2 \leqslant k \leqslant n-1, \varphi \in D(\mathcal{M}, \mathcal{N})$ and $P \in E^{m}$, let us set

$$
\|d \varphi(P)\|_{k}=\max _{\substack{v_{1}, v_{2}, \ldots, v_{k} \in R^{m} \\ \operatorname{vol}\left(v_{1}, v_{2}, \ldots, v_{k}\right) \neq 0}} \frac{\operatorname{vol}\left(d \varphi(P)\left(v_{1}\right), d \varphi(P)\left(v_{2}\right), \ldots, d \varphi(P)\left(v_{k-1}\right), d \varphi(P)\left(v_{k}\right)\right)}{\operatorname{vol}\left(v_{1}, v_{2}, \ldots, v_{k-1}, v_{k}\right)}
$$

where the symbol $\operatorname{vol}\left(w_{1}, w_{2}, \ldots, w_{k-1}, w_{k}\right)$ denotes the $k$-dimensional volume of the $k$-simplex defined by the vectors $w_{1}, w_{2}, \ldots, w_{k-1}, w_{k}$ in $\mathbf{R}^{m}$. With this notation the function $\Theta_{k}((\mathcal{M}, \mathcal{N}, \varphi))=\log \left(\sup _{P \in E^{m}}\|d \varphi(P)\|_{k} \cdot \sup _{Q \in E^{m}}\left\|d \varphi^{-1}(Q)\right\|_{k}\right)$ satisfies the above-mentioned conditions, if we set $\Omega=\Sigma_{n}$ and define $\mathcal{M}_{R} \mathcal{N}$ if and only if there exists a sequence $\left\{\varphi_{i}\right\}_{i \in N}$ of diffeomorphisms in $D(\mathcal{M}, \mathcal{N})$ such that $\lim _{i \rightarrow+\infty} \Theta_{k}\left(\left(\mathcal{M}, \mathcal{N}, \varphi_{i}\right)\right)=0$ (we shall not prove here this statement and the fact that $\mathcal{R}$ is an equivalence relation). The distance $\sigma_{k}$ that we can obtain by using $\Theta_{k}$ is interesting because it corresponds logically to $\sigma$ in the study of $L$-homotopy in degree $k$ (see [5], Remark 3.5). It is easy to verify that for $\mathcal{M}, \mathcal{N} \in \Sigma_{n}$ we have $k \cdot \sigma([\mathcal{M}],[\mathcal{N}]) \geqslant \sigma_{k}([\mathcal{M}],[\mathcal{N}])$.

\section{REFERENCES}

[1] L.V. Ahlfors, Lectures on quasiconformal mappings (Van Nostrand, Princeton, New Jersey, 1966).

[2] L. Bers, 'Uniformization, moduli and Kleinian groups', Bull. London Math. Soc. 4 (1972), 257-300.

[3] L. Cesari, Surface area (Ann. of Math. Stud., Princeton Univ. Press, 1956).

[4] N. Dunford and J.T. Schwartz, Linear operators (Interscience, New York, 1958).

[5] P. Frosini, 'Metric homotopies' (to appear).

[6] A. Jackson, 'Geometry-Supercomputer Project inaugurated', Notices Amer. Math. Soc. 35 (1988), 253-255.

[7] A.N. Kolmogorov and S.V. Fomin, Elements of the theory of functions and functional analysis (Graylock Press, Rochester, New York, 1957).

[8] H.L. Royden, 'Automorphisms and isometries of Teichmüler spaces', in Advances in the Theory of Riemann Surfaces, pp. 369-383 (Princeton University Press, New York, 1971).

Istituto Matematico "Ulisse Dini"

Universiti di Firenze

Viale Morgagni 67/a

50134 Firense

Italy 